\title{
Rosetta swing-by at Mars - an analysis of the ROMAP measurements in comparison with results of 3-D multi-ion hybrid simulations and MEX/ASPERA-3 data
}

\author{
A. Boesswetter ${ }^{1}$, U. Auster ${ }^{2}$, I. Richter ${ }^{2}$, M. Fränz ${ }^{4}$, B. Langlais ${ }^{5}$, S. McKenna-Lawlor ${ }^{6}$, S. Simon ${ }^{1}$, U. Motschmann ${ }^{1,3}$, \\ K. H. Glassmeier ${ }^{2}$, N. J. T. Edberg ${ }^{7}$, and R Lundin ${ }^{8}$ \\ ${ }^{1}$ Institute for Theoretical Physics, TU Braunschweig, Germany \\ ${ }^{2}$ Institute for Geophysics and Extraterrestrial Physics, TU Braunschweig, Germany \\ ${ }^{3}$ DLR, Institute of Planetary Research, Berlin, Germany \\ ${ }^{4}$ Max Planck Institute for Solar System Research, Katlenburg-Lindau, Germany \\ ${ }^{5}$ CNRS, Laboratoire de Planétologie et Géodynamique, Nantes Atlantique Universités, Nantes, France \\ ${ }^{6}$ Space Technology Ltd., National University of Ireland, Maynooth, County Kildare, Ireland \\ ${ }^{7}$ Department of Physics \& Astronomy, University of Leicester, Leicester, UK \\ ${ }^{8}$ Swedish Institute of Space Physics, Kiruna, Sweden
}

Received: 15 May 2008 - Revised: 7 May 2009 - Accepted: 25 May 2009 - Published: 8 June 2009

\begin{abstract}
The Rosetta spacecraft flew by Mars at a distance of $260 \mathrm{~km}$ on 25 February 2007 during a gravity assist manoeuvre. During the closest approach (CA) the lander magnetometer ROMAP was switched on. The dataset taken during this swingby provides insight into the plasma environment around Mars: in addition to a pronounced bow shock crossing Rosetta recorded the signature of the pile up region of draped magnetic field. Also the Rosetta measurements showed signatures of crustal magnetic field anomalies which can be verified by results of a crustal magnetic field model. In order to understand the measured field morphology, multi-ion hybrid simulations were performed. Some of the input parameters for the simulations were obtained from Mars Express (MEX) data which were contemporaneously collected during the Rosetta swingby. These simulations reproduces ROMAP magnetic field measurements and show that the interplanetary magnetic field pointed northward during the encounter. A spectral analysis shows upstream waves ahead of the bow shock and indicates the presence of the magnetic pile-up boundary (MPB). The multi-ion model reproduces the ion fluxes measured by MEX/ASPERA-3 and is in agreement with the measurements to within one order of magnitude.
\end{abstract}

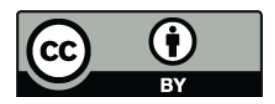

Correspondence to: A. Boesswetter (a.boesswetter@tu-bs.de)
Keywords. Interplanetary physics (Planetary bow shocks) Magnetospheric physics (Plasma waves and instabilities) Space plasma physics (Kinetic and MHD theory)

\section{Introduction}

On 2 March 2004 ESA scientists celebrated the successful launch of the Rosetta spacecraft aboard an Ariane 5 launcher from Kourou in French Guyana to comet 67P/ChuryumovGerasimenko (Glassmeier et al., 2007a). Before the spacecraft reaches its target, a series of swing-by manoeuvres will be accomplished. After the first Earth swingby in March 2005 Rosetta successfully performed a Mars swingby in February 2007. Within the framework of this paper, we focus on plasma and magnetic field observations during the latter swingby. First the data will be discussed and secondly the output from a three-dimensional, multi-species hybrid model will provide a reference to support our analyses. The hybrid approach treats the electrons of the plasma as a fluid, while the ions are represented by individual particles. Thus, this approximation is capable of incorporating effects like nonMaxwellian velocity distributions and differences between the flow patterns of the involved ion species. These phenomena become of importance when the overall size of the interaction region is comparable to the gyroradii of the ions, as is the case at Mars.

Published by Copernicus Publications on behalf of the European Geosciences Union. 
Several hybrid simulations for Mars (Brecht, 1997; Kallio and Janhunen, 2002; Bößwetter et al., 2004, 2007; Modolo et al., 2005) were made in the last ten years. Bößwetter et al. (2004) and Brecht and Ledvina (2006) stated the importance of the temperature of heavy ion plasma for covering the real situation. Modolo et al. (2006) included multiple ionization processes self-consistently in their model and analyzed the influence of the solar EUV flux on the positions of the bow shock and the MPB. Kallio et al. (2006) simulated escaping planetary ions using a hybrid model and found many qualitative and quantitative similarities with the observations made by the Automatic Space Plasma Experiment with Rotating Analyzer (ASPERA) instrument on the Phobos-2 Mission to Mars as well as by ASPERA-3 on MEX. They concluded that the acceleration of planetary ions by the convective electric field associated with the flowing plasma is the key acceleration mechanism for the escaping ions observed at Mars. MHD models do not include the effects of finite ion gyroradii. However, these models have a much higher spatial resolution than kinetic models. Liu et al. (2001) and Ma et al. (2002) showed in their MHD studies good agreement of the positions of the bow shock and the ionopause measured aboard MGS. Ma et al. (2002) demonstrated that crustal magnetic fields did not cause major distortions in the bow shock, although certainly they had an important effect within the magnetosheath. In a recent MHD multi-species model by Ma and Nagy (2007), ion escape fluxes from Mars were calculated for different nominal solar wind, solar cycle and crustal field orientation conditions.

During the Mars swingby the ROMAP instrument aboard Rosetta was switched on at closest approach (CA) for several hours to collect high resolution magnetic field data. This instrument was designed to study the local magnetic environment of the mission's destination comet. Qualitatively it is expected that the plasma interaction of ChuryumovGerasimenko will show some resemblance to the Martian plasma interaction. While both bodies are surrounded by a mainly solar-UV-induced ionosphere, we expect that the Churyumov-Gerasimenko as well as Mars do not possess a substantial magnetic field. Therefore, the solar wind is decelerated upstream of the obstacle, leading to the formation of a bow shock. In both cases the supersonic solar wind is expected to be separated from the ionospheric heavy ion population by an ion composition boundary, as initially reported by Breus et al. (1991) and Sauer et al. (1994). The physics of this boundary layer have already been extensively studied within the framework of numerical models such as the approaches by Sauer and Dubinin (2000), Bößwetter et al. (2004), Simon et al. (2007a), and Bößwetter et al. (2007). Furthermore the interaction leads to the formation of a magnetic pile-up region (MPR) on the ramside of the obstacles. Since the physical properties of these scenarios have been discussed extensively in several other papers (Bagdonat and Motschmann, 2002a,b; Bagdonat et al., 2004; Bößwetter et al., 2004; Simon et al., 2006, 2007b; Bößwetter et al.,
2007) we will not go into the details here.

While our hybrid model has already been successfully applied to reproduce plasma observations near Mars, the approach described here is new in several respects: Our preceding studies did not consider the multi-ion nature of the Martian ionospheric and exospheric plasma. The improved model used in this study considers a multi-component ionosphere consisting of five different ion sources. We did not include the crustal magnetic field into this model version. However, our analysis of the ROMAP data take into account the influence of crustal magnetic fields on the magnetic field morphology.

This paper is organized as follows: in Sect. 2, the magnetic field observations of the Rosetta spacecraft are presented and discussed in detail. The influence of local crustal magnetic field anomalies on the Rosetta measurements is discussed. We demonstrate that some of the measured magnetic field signatures can be explained by applying the crustal magnetic field model developed by Langlais et al. (2004). In Sect. 3, the results of our interpretation are validated by means of a three-dimensional hybrid simulation run. The simulation model is described in this section. The input parameters for the numerical scenario are partially obtained from Rosetta measurements. However, since onboard plasma instruments making up the Rosetta Plasma Consortium (RPC) remained offline around CA, no upstream densities and velocities for the impinging solar wind are available from this mission. Therefore, to complete the set of input parameters for the model, we used plasma moments from the MEX mission, which had been engaged in simultaneously collecting plasma data during the time of the Rosetta swingby. The discussion not only focuses on the interpretation of the data, but also on the global topology of the Martian plasma environment within the framework of the multi-species model. Further, we compare global modeling results with the foregoing study (Bößwetter et al., 2007), which considered only a single ionospheric species. The work concludes with a brief summary of our major findings and a view toward future projects.

\section{Rosetta magnetic field measurements}

In this section we present magnetic field data from Rosetta's lander magnetometer ROMAP and from the orbiter magnetometer RPC-MAG and compare the magnetic field signatures obtained near closest approach with a crustal magnetic field model derived from MGS magnetic field data.

Figure 1 shows the projection of Rosetta's trajectory and of the MEX orbit on the Mars Solar Orbital (MSO) coordinate planes. The $x$-axis points towards the sun, the $y$-axis is contrary to the planetary motion, and the z-axis completes the right-handed system pointing towards the north pole of the planet. Rosetta arrived from the undisturbed solar wind regime on the dawn side of the planet reaching an altitude of 

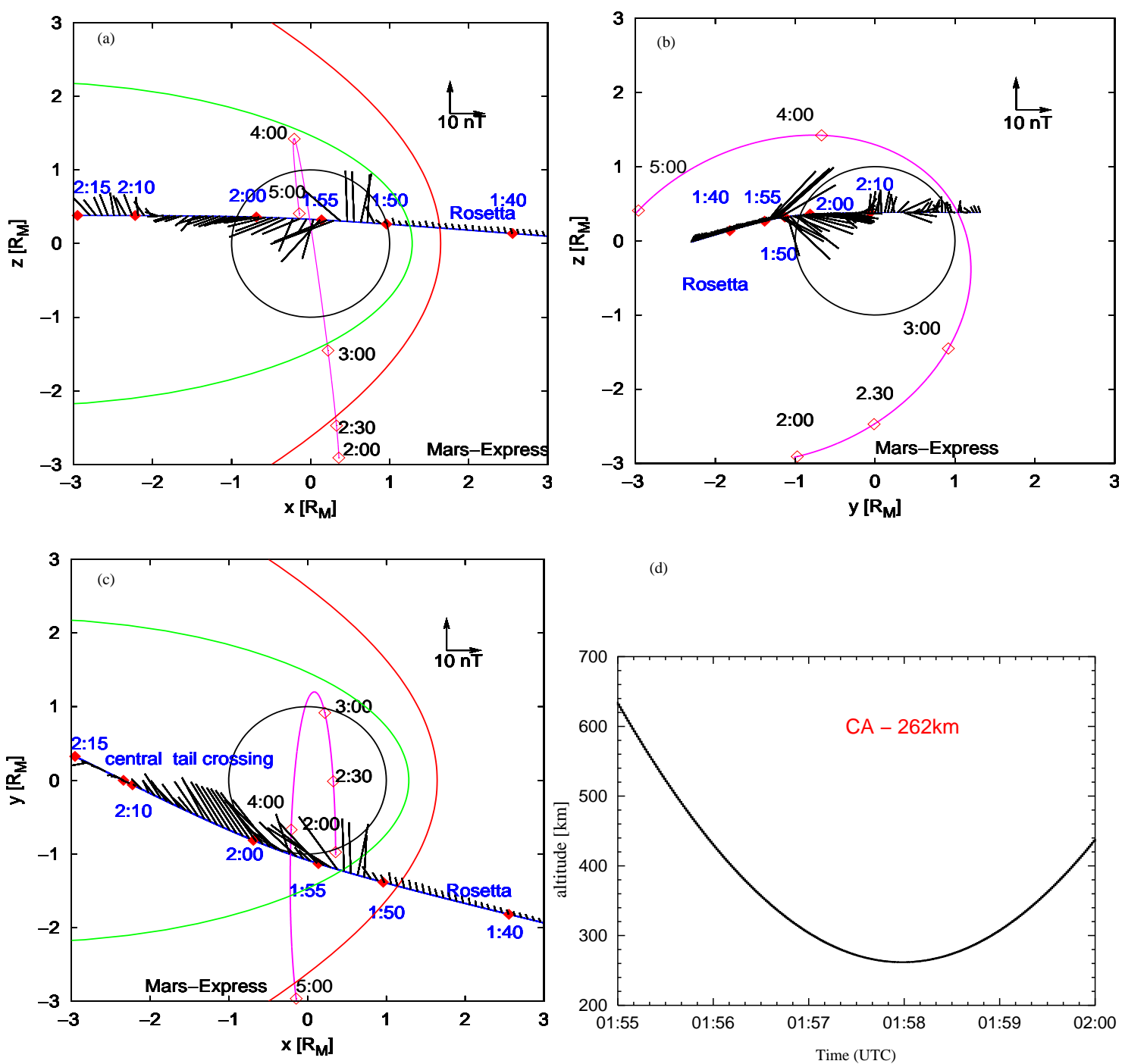

Fig. 1. (a-c) Projections of the trajectory of Rosetta (in blue) and MEX orbit in pink) on the MSO coordinate planes on 25 February 2007. The $\mathrm{x}$-axis points toward the sun, the $\mathrm{y}$-axis points in negative direction of the orbital motion and the $\mathrm{z}$-axis complete the right hand system pointing to the north pole of the planet. Rosetta comes from the undisturbed solar wind regime on the dawn side of the planet and reached an altitude of $260 \mathrm{~km}(\mathrm{CA})$ at around 05:00 LT=01:58 UT, still at the night side. Rosetta's trajectory lays in the equatorial plane, shifted only slightly into the Northern Hemisphere. The orbital plane of MEX is tilted predominantly to the terminator (x-z plane). Additionally magnetic field measurements of ROMAP are presented along Rosetta's trajectory. The average bow shock position by MGS is presented in red and the average MPB position in green (Vignes et al., 2000). (d) Altitude of Rosetta above the Martian surface during the passage of CA.

$262 \mathrm{~km}(\mathrm{CA})$ at around 05:00 Martian local time at the night side. Rosetta's trajectory was located in the equatorial plane, shifted only slightly into the Northern Hemisphere. The orbital plane of MEX is tilted predominantly to the terminator (x-z plane). In addition, magnetic field measurements of ROMAP are presented along Rosetta's trajectory. The average bow shock position measured by MGS is denoted in red and the average MPB position is represented by the green line (Vignes et al., 2000).

\subsection{ROMAP data}

During the close swingby of Mars the Rosetta lander magnetometer instrument ROMAP collected high resolution data $(64 \mathrm{~Hz})$ in a time interval of about one hour, centered around closest approach at 01:58 UT. A detailed description of the instrument is provided by Auster et al. (2007).

The lander magnetometer ROMAP detected the Martian bow shock at 01:51 UT. After leaving the shock front, the spacecraft entered the set of highly draped magnetic field lines at the Martian ram side, which is often referred to 

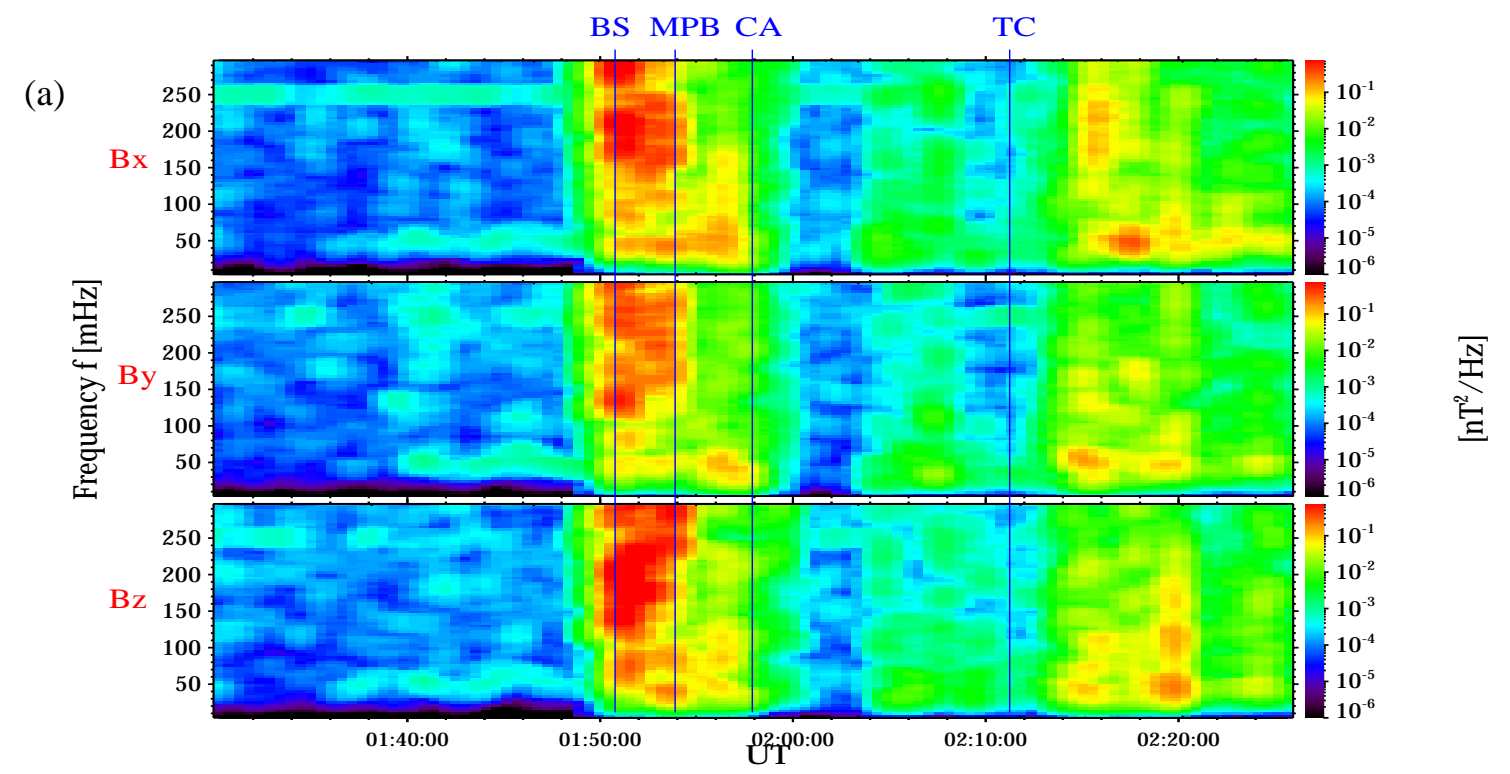

(b)

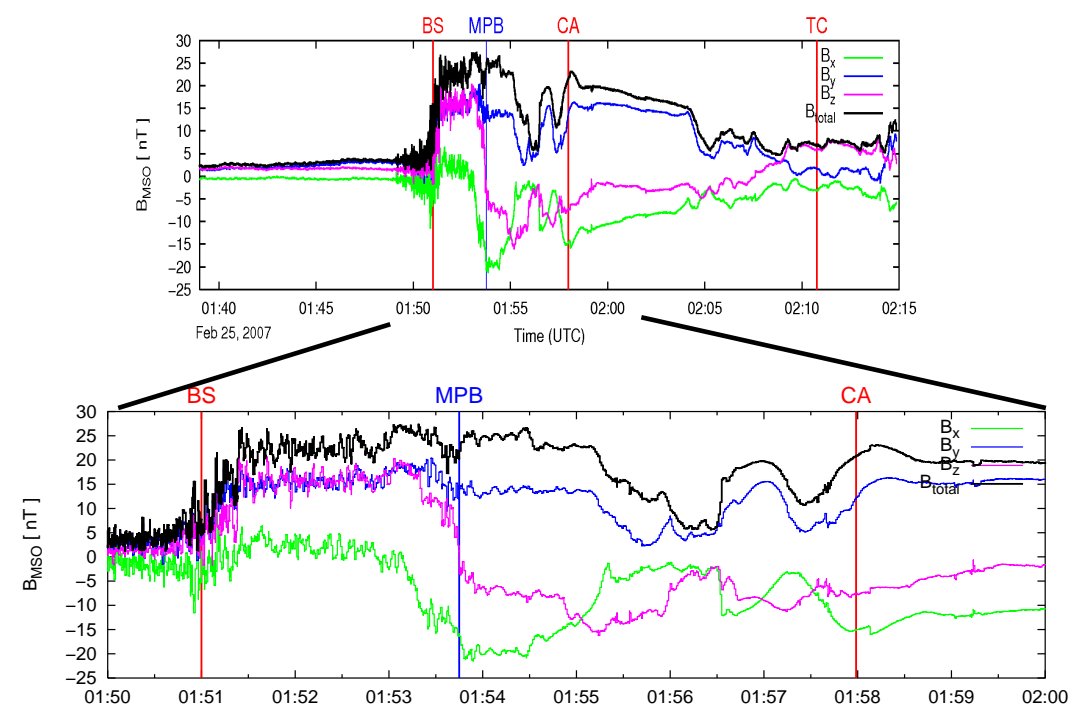

Fig. 2. Magnetic field data measured by ROMAP, (a) Dynamic Frequency Spectra, (b) data in MSO coordinates, (c) detailed plot of data. About $10 \mathrm{~min}$ before the bow shock is crossed, ROMAP measures low frequency waves between $40-70 \mathrm{mHz}$, proton upstream waves which were also reported by Brain (2004). After detecting a fore shock region at around 01:49 UT, ROMAP crosses the bow shock at 01:51 UT where the magnetic field jumps quite strongly from $4 \mathrm{nT}$ in the solar wind to around $10 \mathrm{nT}$ in fore shock region and to $25 \mathrm{nT}$ behind the bow shock. The fluctuations appear in all frequency ranges. At 01:53:45 UT Rosetta enters the MPB, where fluctuations are clearly reduced. At 01:58 UT Rosetta reaches its CA with $262 \mathrm{~km}$ above surface. A minute before, already under $300 \mathrm{~km}$ above surface, fluctuations by weak crustal magnetic fields are measured (see also Fig. 4). Behind the tail crossing (TC) fluctuations again are increased in all three components. The signal at around $250 \mathrm{mHz}$ is a spacecraft-generated artifact. Note that the offsets of the three components could not be determined exactly. However, the data are coherent, especially the clear negative $B_{x}$ component in the magnetosheath leads to the explanation that IMF had a strong $B_{z}$ component (see Fig. 10). This behavior could also be verified by hybrid simulation results (see Fig. 9).

as the MPR, as e.g. discussed by Bertucci et al. (2003), Bertucci et al. (2005a), and Brain et al. (2006) and modeled by Bößwetter et al. (2004) and Modolo et al. (2006).

As displayed in Fig. 2, the foreshock region is clearly visible in the data collected during the Rosetta approach. The power spectrum of the magnetic field indicates the presence of wave activity of up to $10^{-3} \mathrm{nT}^{2} / \mathrm{Hz}$ in all three components from 40-70 $\mathrm{mHz}$ between 01:36 UT and 01:50 UT. At 01:48 UT, a peak frequency of $60 \mathrm{mHz}$ was measured in all three components. The proton gyrofrequency in a background field of $B=4 \mathrm{nT}$ is $f_{P}=e B /\left(2 \pi m_{p}\right)=61 \mathrm{mHz}$. These waves at the local proton gyrofrequency (PCWs) are low 
amplitude waves $(\approx 0.15 \mathrm{nT})$, and they have been described by Russell et al. (1990) using ASPERA data measured on Phobos-2 and by Brain et al. (2002) using MGS magnetic field data. As Brain et al. (2002) stated, these waves were not observed at larger subsolar distances than $3 R_{M}$ from Mars. The first signature of the upstream waves in ROMAP data was obtained at 01:36 UT, when Rosetta was at a distance of about $2 R_{M}$ from the surface of the planet. Russell et al. (1990) concluded that these left-hand polarized waves were formed by ionization of the Martian hydrogen exosphere upstream of the shock. Barabash et al. (1991) confirmed this conclusion by results from ASPERA, which observed ring distributions of pickup protons from the hydrogen corona. Ring distributions are unstable and generate Alfven waves which can be recorded using a magnetometer.

In the foreshock region the measured magnetic field exhibited minor fluctuations on a time scale of only a few seconds while, simultaneously, the foot of the bow shock was reached where the field magnitude increased from 4 to about $6 \mathrm{nT}$. The upstream side of the bow shock was reached at about 01:51 UT and a sharp increase in the magnetic field magnitude by a factor of about 4, see Fig. 2c was recorded. After this, Rosetta enteres the MPR at 01:54 UT. In the magnetometer data this region becomes manifest through a rotation of the magnetic field vector that was in agreement with previous observations (Bertucci et al., 2005b) as well as numerical modeling attempts (Bößwetter et al., 2004; Modolo et al., 2006). The spectrum in Fig. 2a shows the same behavior: namely reduced wave power in all three components over the middle and high frequency ranges $(100 \mathrm{mHz}$ and above) from 1 to $10^{-2} \mathrm{nT}^{2} / \mathrm{Hz}$ at the location of the MPB. We interpret this behavior to provide an indication of the MPB as Rosetta's trajectory crosses the magnetic configuration in such a way that no jump occured. One minute before closest approach, at around 01:57 UT, Fig. 2c exhibits well defined, sharply pronounced spikes in all three components. As we will show later in detail Rosetta associatively crossed a weak crustal magnetic field region.

Rosetta traversed a magnetically very quiet region after passing the CA. The wave power continued to decrease over the whole frequency range from $10^{-2} \mathrm{nT}^{2} / \mathrm{Hz}$ to $10^{-4} \mathrm{nT}^{2} / \mathrm{Hz}$. On crossing the Mars tail-midnight line (TC) at around 02:11 UT, wave power again increased to values between $10^{-2} \mathrm{nT}^{2} / \mathrm{Hz}$ and $10^{-1} \mathrm{nT}^{2} / \mathrm{Hz}$.

As discussed by Edberg et al. (2009), Rosetta remained within the thermalized plasma of the Martian magnetosheath for about two hours after CA, before crossing the bow shock again and exiting to the solar wind at 04:04 UT. The present authors discuss in addition the possibility of a second bow shock crossing due to an expansion of the Martian magnetosphere in the outbound region of the swingby.

\subsection{RPC-MAG data}

The second magnetometer RPC-MAG (Glassmeier et al., 2007b), located on the orbiter, consists of two sensors, namely an inboard (IB) and an outboard (OB) sensor. This configuration provides a method to deduce and remove spacecraft-generated magnetic field disturbances from the data set (such as currents on the spacecraft and temperature changes) Fig. 3 features the magnetic field data measured by the RPC-MAG outboard sensor for the period 23-25 February 2007. Both the inboard and the outboard sensor showed similar signatures during this interval. During a period between 24 February at 17:00 UT and 25 February at 08:00 UT discrepancies were found between the data collected by both sensors. We neglect this latter period in our analysis and determine a mean background magnetic field outside the Martian magnetosheath of $\boldsymbol{B}=(0,0,4) \mathrm{nT}$ as input parameter for the hybrid simulations discussed later. All magnetic field components showed quite strong disturbances ranging from $-15 \mathrm{nT}$ to $+15 \mathrm{nT}$ two days before and after the CA. This behaviour demonstanted non-stationary solar wind conditions. During the CA, RPC-MAG and all the other instruments of the orbiter payload were switched off to conserve power.

\subsection{Crustal magnetic field}

An early idea of remanent crustal magnetism at Mars was given by Curtis and Ness (1988). Moehlmann (1992) found first hints concerning a crustal magnetic field. Thereafter, MGS detected a local crustal magnetization, mainly above the Southern Hemisphere (Acuña et al., 1999; Connerney et al., 1999). Its amplitude at $200 \mathrm{~km}$ altitude varied between $\pm 650 \mathrm{nT}$. At the surface, its magnitude may reach several thousand $\mathrm{nT}$.

Several attempts were made to model the local magnetic field anomalies at Mars using different techniques and datasets. Purucker et al. (2000) used low altitude, purely radial, preliminary binned MGS magnetic observations to produce a magnetic field map at constant altitude. An improved version of the original Purucker model used calibrated data. It corrected a small numerical error which resulted in evidence for a global magnetic field, but the resulting model was still incomplete, due to the far-from-complete low-altitude data coverage. Other studies by Arkani-Hamed (2002) and Cain et al. (2003) employed with the commonly used spherical harmonic method of Gauss (1839). An updated model by Arkani-Hamed (2002) used three components of low and high altitude measurements to produce a spherical harmonic model up to degree 50. Cain et al. (2003) derived another spherical model up to degree 90. Another model was published by Langlais et al. (2004), who used multiple sets of MGS magnetometer data measured during different phases from 1997-2001 in different altitude ranges. They considered Equivalent Source Dipoles (ESD) located on an equidistant icosahedral mesh beneath the Martian surface. Their 


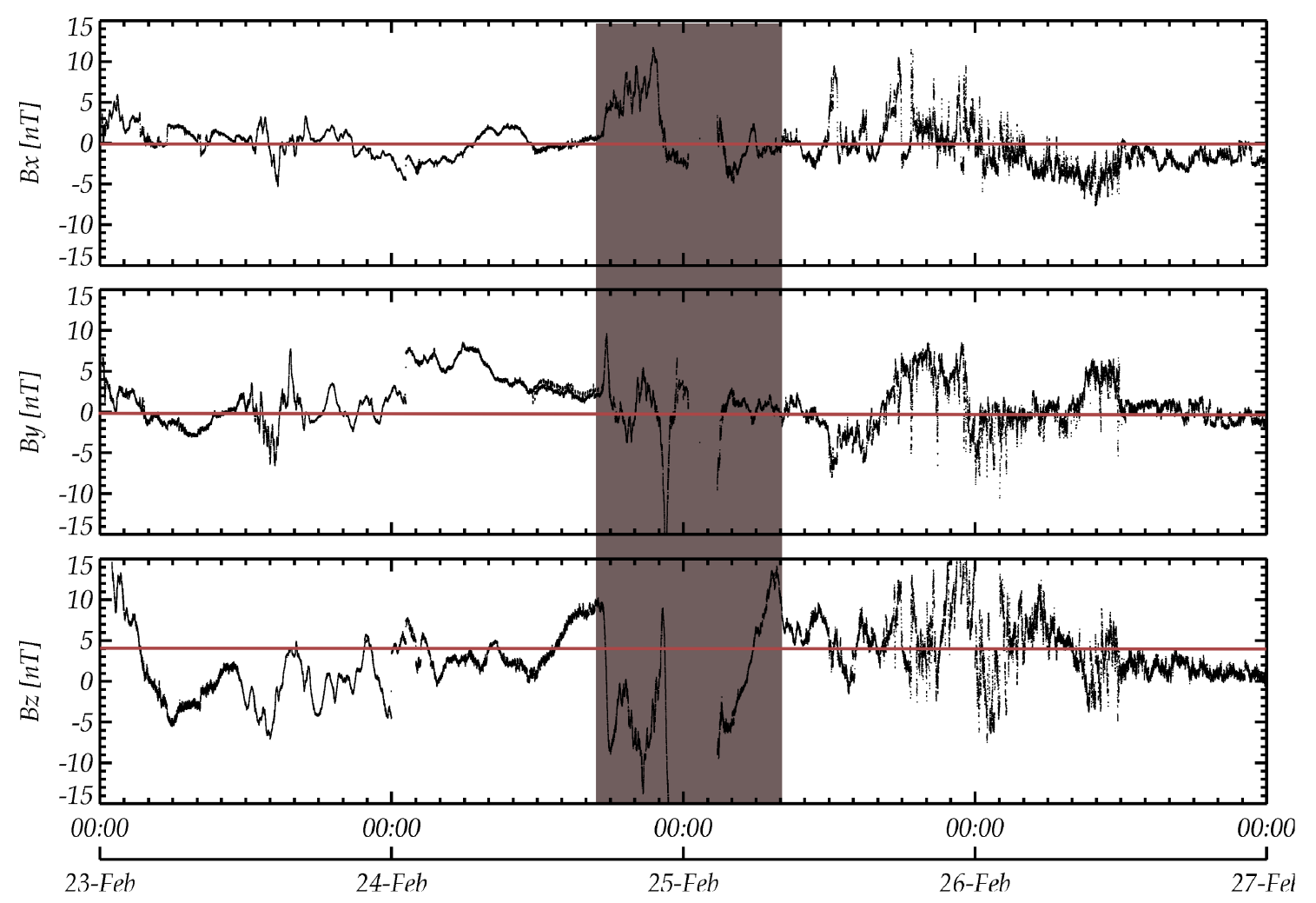

Fig. 3. Magnetic field data in MSO coordinates measured by RPc-MAG outboard sensor on 23-25 February 2007. Both the inboard and the outboard MAG sensor show the same trend. Only the dark shaded period between 24 February at 17:00 UT and 25 February at 08:00 UT exhibits a different behavior of both sensors. This difference is to be regarded as an indication of disturbances caused by the spacecraft like e.g. currents and temperature changes. We neglect this period for the determination of a mean background magnetic field outside the Martian magnetosheath. Thus as input parameter for the hybrid simulation we use $\boldsymbol{B}=(0,0,4) \mathrm{nT}$.

dipole mesh has a mean spacing of $173 \mathrm{~km}$, or $2.91^{\circ}$ at the equator. We provide below a comparison with this sophisticated model.

Figure 4 shows ROMAP magnetic field data and the predictions of the latest crustal magnetic field model by Langlais et al. (2004). Here we use planetary Cartesian (pc) coordinates which are defined as follows: the positive $\mathrm{x}$ and positive y-direction define the equatorial plane and are respectively directed toward $0^{\circ}$ and $90^{\circ}$ Eastern longitude. The positive z-direction is oriented toward the geographical North pole. The ROMAP data are detrended, which means that we subtracted all low frequency information below $6 \mathrm{mHz}$ from the data. High frequency disturbances from the shocked solar wind remained after the bow shock crossing. However, most contributions in the data near CA emerged in the vicinity of the crustal field anomalies at around 01:56:30 UT and 01:58:40 UT (see Fig. 1d). During this time period Rosetta was below $300 \mathrm{~km}$ near the Martian terminator. This altitude range was below that of the MPB, which is typically located at altitudes of $1200-1600 \mathrm{~km}$ at the terminator (Trotignon et al., 1996; Vignes et al., 2000). Rosetta was still on the night side of the planet. Disturbances by the sunlight did not appear. These good conditions allow a comparison to be made between the measured magnetometer data and a crustal field model. The model results used here, which was provided by Langlais et al. (2004), predicted a weak magnetic field strength below $10 \mathrm{nT}$, which is in agreement with the ROMAP data. As can be seen in Fig. 4, nearly every peak in this region can be reproduced by the crustal model. By subtracting the low frequency trend from the model data, the amplitude of the crustal signature also fits quite well the detrended ROMAP measurements.

\section{Hybrid multi-ion simulation}

The numerical investigations are based on the hybrid code developed by Bagdonat and Motschmann (2002a). The present multi-ion version of the code is an update of the version that has already been successfully applied to the solar wind interaction with comets (Bagdonat et al., 2004; Motschmann and Kührt, 2006), magnetized asteroids (Simon et al., 2006) as well as to the plasma environment of Mars 

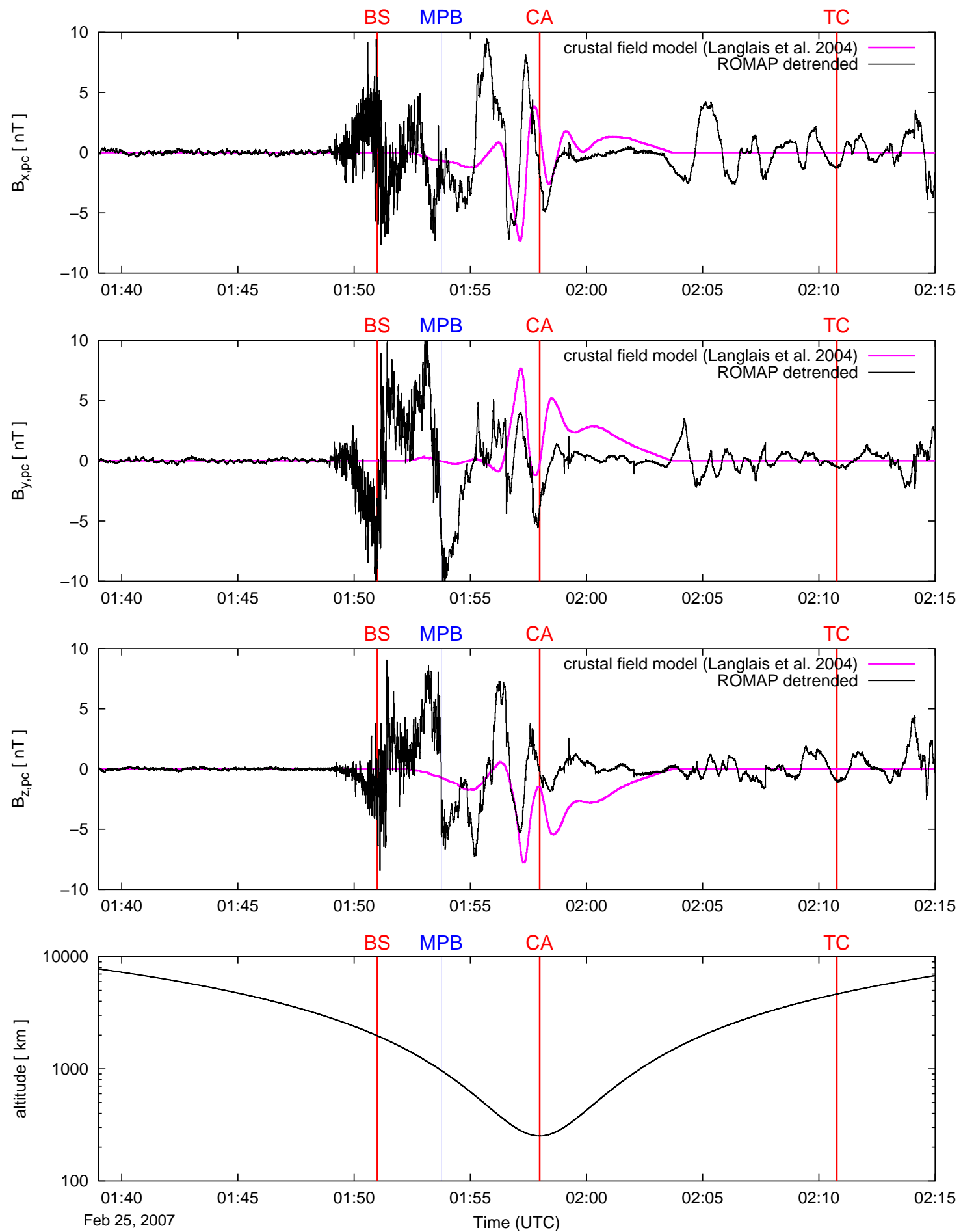

Fig. 4. Detrended ROMAP data in planetary Cartesian coordinates in comparison with the predictions by Langlais et al. (2004). All peaks in the ROMAP data can be predicted by the crustal model.

(Bößwetter et al., 2004) and Titan (Simon et al., 2007b; Simon et al., 2008). This new version includes the three main ionospheric ion species, namely $\mathrm{CO}_{2}^{+}, \mathrm{O}_{2}^{+}$and $\mathrm{O}^{+}$, as well as the dominant exospheric ions $\mathrm{O}^{+}$and $\mathrm{H}^{+}$. For compari- son, the original code contained only one planetary ion component representing the ionospheric and exospheric oxygen ions. 


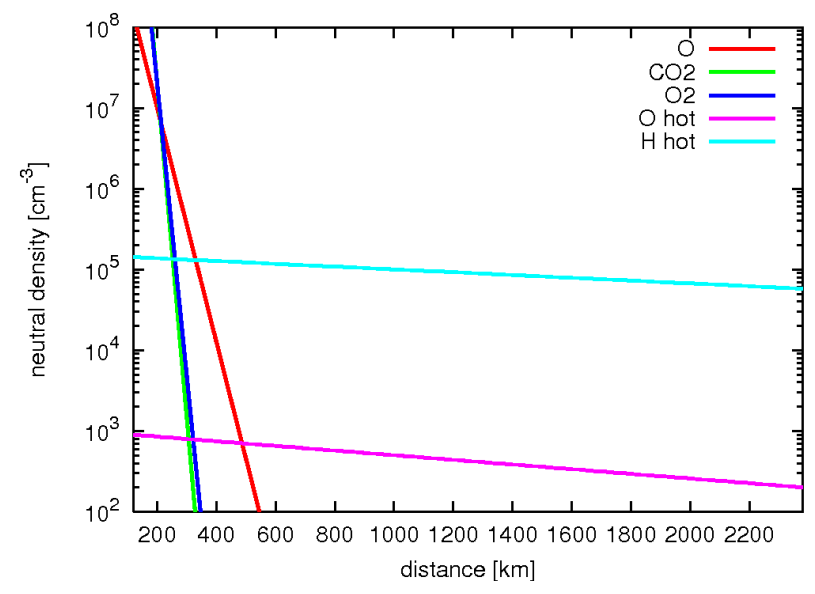

Fig. 5. Neutral density per species used in hybrid simulations. The atmospheric profiles were taken from Krasnopolsky (2002), Exospheric hydrogen and oxygen profiles from Chaufray et al. (2007) for solar minimum conditions. Note the two orders of magnitude denser exospheric hydrogen density than the oxygen density. Ions are produced by photoionization and charge exchange in the range $200 \mathrm{~km}-2 R_{M}$ above the surface.

\subsection{Model description}

In the hybrid approximation, the electrons are modeled as a massless charge-neutralizing fluid, whereas the ions are treated as individual particles. Here we do not go into a detailed description of the code which can be found in Bößwetter et al. (2004). In what follows, only the dynamic equations of the model will be presented.

- Equation of motion for individual ions:

$$
\frac{\mathrm{d} \boldsymbol{v}_{s}}{\mathrm{~d} t}=\frac{q_{s}}{m_{s}}\left(\boldsymbol{E}+\boldsymbol{v}_{s} \times \boldsymbol{B}\right)-k_{D} n_{n}\left(\boldsymbol{v}_{s}-\boldsymbol{u}_{n}\right)
$$

where $q_{s}, m_{s}$ and $\boldsymbol{v}_{s}$ respectively denote the charge, mass and velocity of an individual particle of species $s$. $k_{D}$ is a phenomenological and temperature dependent parameter describing the collisions of ions and neutrals, a good mean value for which of $1.7 \times 10^{-9} \mathrm{~cm}^{3} \mathrm{~s}^{-1}$ was given by Israelevich et al. (1999). For example, for proton-oxygen collisions one can calculate for $200 \mathrm{~km}$ above the surface using an ion temperature of $500 \mathrm{~K}$ a value of $1.1 \times 10^{-9} \mathrm{~cm}^{3} \mathrm{~s}^{-1}$. At $400 \mathrm{~km}$ with an ion temperature of around $3000 \mathrm{~K}$ the collision frequency increases only slightly to $2.5 \times 10^{-9} \mathrm{~cm}^{3} \mathrm{~s}^{-1} . n_{n}$ and $\boldsymbol{u}_{n}$ are the number density and bulk velocity of the neutrals. We use $\boldsymbol{u}_{n}=0$.

- Electric field equation:

$$
\boldsymbol{E}=-\boldsymbol{u}_{i} \times \boldsymbol{B}+\frac{(\nabla \times \boldsymbol{B}) \times \boldsymbol{B}}{\mu_{0} e n_{e}}-\frac{\nabla P_{e, \mathrm{sw}}+\nabla P_{e, \mathrm{hi}}}{e n_{e}}
$$

Table 1. Ion production frequencies. The ions are produced by photoionisation. Exospheric ions are additionally produced by charge exchange with solar wind protons.

\begin{tabular}{ll}
\hline & Numerical value \\
\hline $\mathrm{O}_{2}^{+}$ & $0.6 \times 10^{-6} \mathrm{~s}^{-1}$ \\
$\mathrm{CO}_{2}^{+}$ & $0.4 \times 10^{-6} \mathrm{~s}^{-1}$ \\
$\mathrm{O}^{+}$ & $0.1 \times 10^{-6} \mathrm{~s}^{-1}$ \\
$\mathrm{O}_{\text {EXO }}^{+}$ & $0.28 \times 10^{-6} \mathrm{~s}^{-1}$ \\
$\mathrm{H}_{\text {EXO }}^{+}$ & $0.72 \times 10^{-6} \mathrm{~s}^{-1}$ \\
\hline
\end{tabular}

where $\boldsymbol{u}_{i}$ is the mean ion velocity. Since the plasma is assumed to be quasi-neutral, the mean ion density $\left(n_{i}\right)$ is equal to the electron density $\left(n_{e}\right)$. As the electron temperature in the solar wind differs by several orders of magnitude from the electron temperature in the Martian ionosphere (Hanson and Mantas, 1988), two different electron pressure terms $P_{e, \mathrm{sw}}$ and $P_{e \text {,hi }}$ were incorporated into the simulation model. Both electron populations are assumed to be adiabatic, i.e.

$$
P_{e, \mathrm{sw}} \propto \beta_{e, \mathrm{sw}} n_{\mathrm{sw}}^{\kappa} \quad \text { and } \quad P_{e, \mathrm{hi}} \propto \beta_{e, \mathrm{hi}} n_{\mathrm{hi}}^{\kappa}
$$

An adiabatic exponent of $\kappa=2$ was used (Bößwetter et al., 2004).

- Magnetic field equation: For the time evolution of the magnetic field one obtains from Faraday's law

$$
\frac{\partial \boldsymbol{B}}{\partial t}=\nabla \times\left(\boldsymbol{u}_{i} \times \boldsymbol{B}\right)-\nabla \times\left[\frac{(\nabla \times \boldsymbol{B}) \times \boldsymbol{B}}{\mu_{0} e n_{e}}\right] .
$$

The electron pressure terms do not occur in this equation, because of $\nabla \times \nabla \mathrm{P}=0$ applied to $P_{e, \text { sw }}$ and $P_{e, \mathrm{hi}}$.

For the simulations of the Rosetta swingby we used a Cartesian simulation grid with a grid resolution of $160 \mathrm{~km}$ per cell.

The Martian atmosphere was modeled as a spherically symmetric gas cloud around Mars consisting of five atomic species: namely the atmospheric ones $\mathrm{CO}_{2}, \mathrm{O}_{2}$ and $\mathrm{O}$ and exospheric, hot, hydrogen and oxygen. Figure 5 shows the radial density distribution for the ionospheric and exospheric exponential profiles. The exospheric profiles were taken from (Chaufray et al., 2007), the atmospheric profiles were taken from Krasnopolsky (2002) for solar minimum conditions.

Assuming a constant solar UV radiation yields the dayside ion production function $q(r, \chi)$ in the form of a Chapman layer for atmospheric ions which depends on both the altitude $r$ above the surface and the solar zenith angle $\chi$. The nightside production profile is assumed to be independent of the solar zenith angle. It is set to an altitude-dependent value of $q\left(r, \chi=87^{\circ}\right)$. This yields a peak ion production rate 


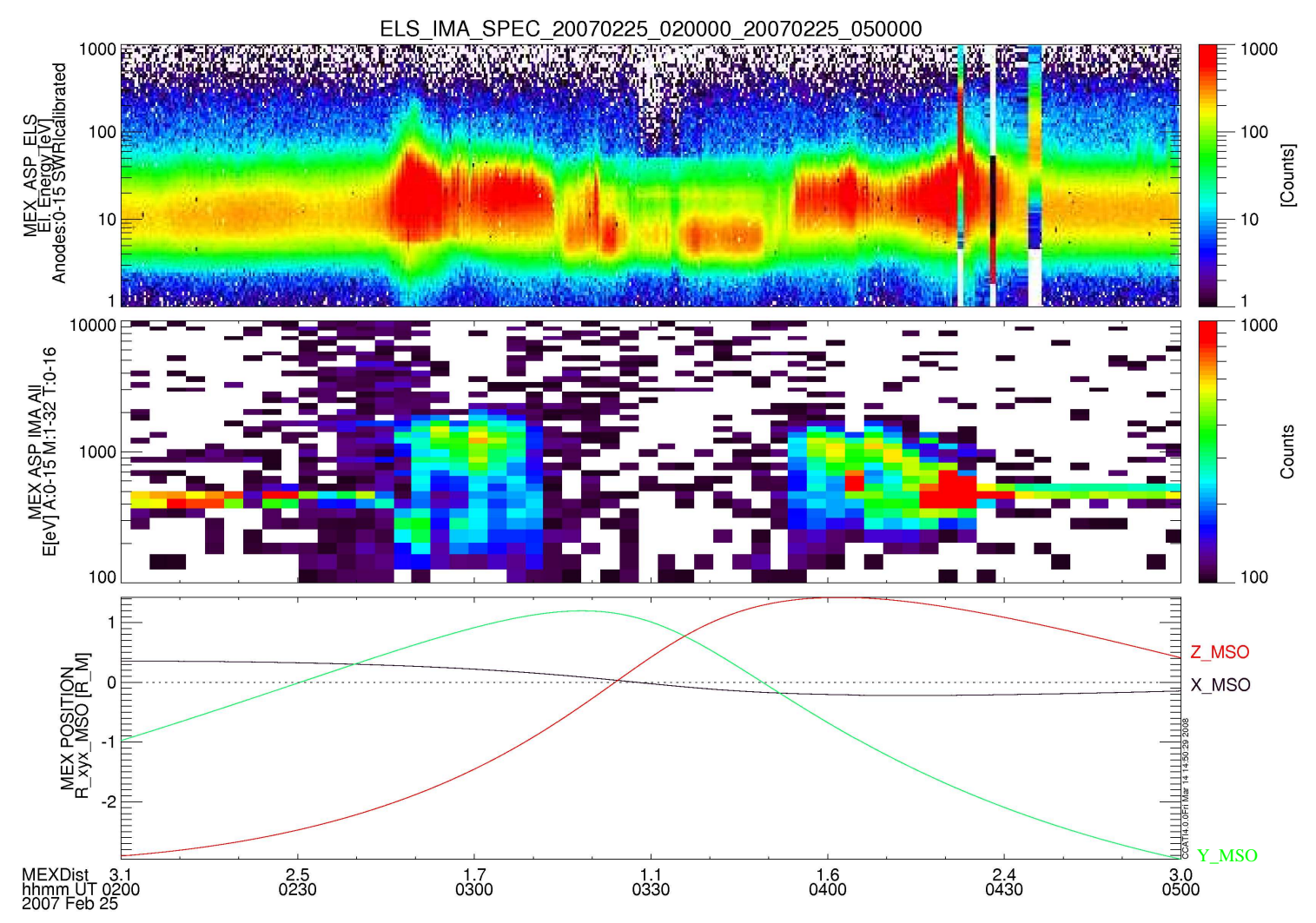

Fig. 6. Electron and ion spectra measured by ELS and IMA sensors onboard MEX/ASPERA-3 from 02:00 UT (only two minutes after Rosetta's closest approach) till 05:00 UT. Additionally, the coordinates of the MEX orbit in MSO coordinates are given at the bottom panel. MEX enters the foreshock region, the bows shock, and the ICB/MPB at 02:45 UT, 02:48 UT, and 03:14 UT, respectively. At the ICB/MPB location the intensity in the ion spectrum falls sharply over the whole energy range. IMA could only measure solar wind protons and He ions. The ionopause is crossed at 03:22 UT, afterwards low energetic electrons at around $5 \mathrm{eV}$ and atmospheric photoelectrons at around $20 \mathrm{eV}$ are measured. During the outbound path data gaps in ELS occur. See Fig. 8 for the from the spectra derived data.

which is about $10 \%$ of the dayside value. Exospheric ions are produced by UV radiation as well, charge exchange reactions with solar wind protons. The charge exchange rate with solar wind ions can be calculated as $n_{s w} * u_{s w} * \sigma$ where $n_{s w} * u_{s w}$ is the background solar wind flux. $\sigma$ represents the cross section parameter. We used $\sigma_{O}=8 \times 10^{-16} \mathrm{~cm}^{2}$ and $\sigma_{H}=3 \times 10^{-15} \mathrm{~cm}^{2}$ from Stebbings et al. (1964). All heavy ions were inserted into the simulation box above the altitude of $200 \mathrm{~km}$ which is the exobase altitude at Mars.

Table 1 contains the relevant ion production frequencies.

Any ion hitting the so-called "Inner Boundary" at an altitude of $150 \mathrm{~km}$ above the planetary surface was removed from the simulation. No boundary conditions were imposed on the electromagnetic fields, i.e. the equations for $\boldsymbol{E}$ and $\boldsymbol{B}$ were solved outside as well as inside the obstacle. An artifical inner density was assumed in order to match the surrounding ionospheric heavy ion density at the subsolar point as well as to avoid electric fields arising from density gradients.

\subsection{Selection of input parameters using MEX data}

The MEX spacecraft was orbiting Mars near the terminator plane with closest approach at 03:35 UT when Rosetta performed its swing by manoeuvre at Mars. At 02:00 UT MEX was still in the solar wind, when Rosetta passed the upper ionospheric layers of Mars. Both spacecraft were at a distance of only $3.3 R_{M}$. As Fig. 1 shows, MEX came from the Southern Hemisphere into the Martian magnetosheath and left it on the dawn side of the planet. During this orbit the plasma sensors of the ASPERA-3 instrument onboard MEX measured electron and ion data. MEX does not carry a magnetometer, so that no magnetic field data are available to cross-calibrate the datasets.

The ELS and IMA sensors of the ASPERA-3 experiment onboard MEX supported the computation of electron as well as ion moments. In this paper we use electron data from the ELS sensor with $4 \mathrm{~s}$ time resolution and ion data from the IMA sensor with $192 \mathrm{~s}$ time resolution. IMA provides ion measurements in the energy range between 0.02 and 

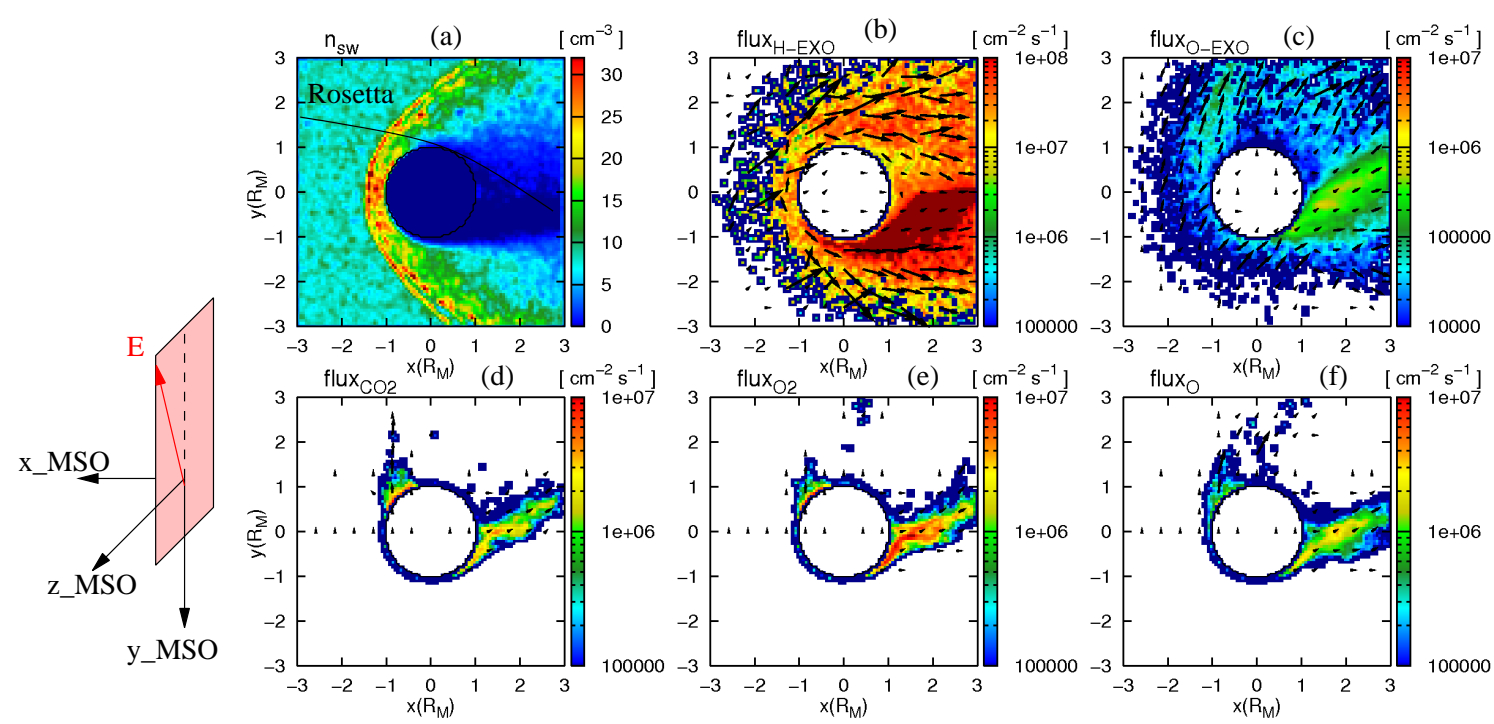

Fig. 7. Contour plots in the equatorial plane from a hybrid simulation. Solar wind density (a) and ion flux values (b-f) are presented. Panel (a) shows the solar wind density $\left(n_{s w}\right)$, panel (b) the particle flux of $\mathrm{H}^{+}$ions originating from the hydrogen exosphere (flux $\mathrm{H}-\mathrm{EXO}$ ), panel (c) shows the particle flux of $\mathrm{O}^{+}$ions originating from the oxygen exosphere (flux $\mathrm{O}-\mathrm{EXO}$ ), panels (d), (e) and (f) show the particle flux of $\mathrm{CO}_{2}^{+}, \mathrm{O}_{2}^{+}$and $\mathrm{O}^{+}$ions, respectivally (flux $\mathrm{CO} 2$, flux $\mathrm{O}_{2}$, flux $\mathrm{O}$ ). The convective electric field vector lays in the plane $\mathrm{y}-\mathrm{z}-\mathrm{plane}$. The projection of Rosetta's trajectory is shown in panel (a). In front of Mars a bow shock is formed, which shows a two-shocklet structure. The solar wind gets strongly mass-loaded by ionospheric and exospheric heavy ions (b-f). Exospheric oxygen ions are picked up by the solar wind electric field (c). In the wake region of the planet plasma cloud structures are detached from the ionosphere and are dragged away into the tail $(\mathrm{d}-\mathrm{f})$.

Table 2. Input parameters for the simulation.

\begin{tabular}{lll}
\hline Parameter & Symbol & Numerical value \\
\hline Solar wind density & $n_{s w}$ & $8.0 \mathrm{~cm}^{-3}$ \\
Solar wind velocity & $u_{s w}$ & $290 \mathrm{~km} / \mathrm{s}$ \\
Dynamical SW pressure & $M_{p}$ & $1.12 \mathrm{nPa}$ \\
Alfvenic Mach Number & $M_{A}$ & 9.39 \\
Background magnetic field & $B_{s w}$ & $4(-0.09,0.15,0.98) \mathrm{nT}$ \\
Proton temperature & $T_{p}$ & $50000 \mathrm{~K}=4.3 \mathrm{eV}$ \\
Electron temperature & $T_{e}$ & $200000 \mathrm{~K}=17.3 \mathrm{eV}$ \\
Ionospheric electron temp. & $T_{e, h i}$ & $3000 \mathrm{~K}=0.26 \mathrm{eV}$ \\
\hline
\end{tabular}

$30 \mathrm{keV} / \mathrm{q}$ for the main ion components with charge number $q$. ELS provides electron measurements in the energy range between 0.01 and $20 \mathrm{keV}$. A detailed description of the instrument is given in Barabash et al. (2006).

Figure 6 shows the electron and ion spectra measured by ASPERA-3. We derived densities and velocities from these spectra to obtain input parameter for the hybrid simulation. MEX entered the foreshock region at 02:45 UT, where the electrons become more and more thermalized. It crossed the bow shock at 02:48 UT and entered the ion composition boundary (ICB) at 03:14 UT. At this boundary IMA shows a sharp decrease in the proton flux. The ionopause was reached at 03:22 UT, where only low energetic electrons are measured. Outbound MEX crossed the ionopause, the ICB, the bow shock, and the foreshock at, respectively, 03:49 UT, 03:54 UT, 04:25 UT, and 04:32 UT. Data gaps occurred in the ELS data during the outbound bow shock crossing. Also, heavy planetary ions could not be observed as the corresponding mode of the instrument was not in operation during the swingby. The most reliable parameter from the IMA data was the proton velocity which was approximately $290 \mathrm{~km} / \mathrm{s}$. The solar wind density varied from 2 up to $10 \mathrm{~cm}^{-3}$, depending on the procedure adopted to fit the ELS spectra. IMA gave a value of about $1-3 \mathrm{~cm}^{-3}$. We present results from a simulation run using an upstream density of $n_{s w}=8.0 \mathrm{~cm}^{-3}$ and a velocity of $u_{s w}=290 \mathrm{~km} / \mathrm{s}$, which fit the MEX plasma measurements. The solar wind density and velocity in Table 2 are typical for slow streaming solar wind type. Measurements by Helios 1 and 2 (Schwenn, 1990) showed that this solar wind type is characterised by a high density $\left(\approx 8.3 \mathrm{~cm}^{-3}\right.$ at earth orbit) and low velocity ( $\approx 327 \mathrm{~km} / \mathrm{s}$ at earth orbit). To obtain the orientation of the IMF in the equatorial plane, we use an IMF angle of $120^{\circ}$ with respect to the MarsSun line (duskward from Mars). This angle was calculated, using the Hakamada-Akasofu-Fry version 2 model/HAFv. 2 (McKenna-Lawlor et al., 2005), to lie within the approximate range $120^{\circ}-160^{\circ}$ on 25 February 2007 at 02:00 UT 

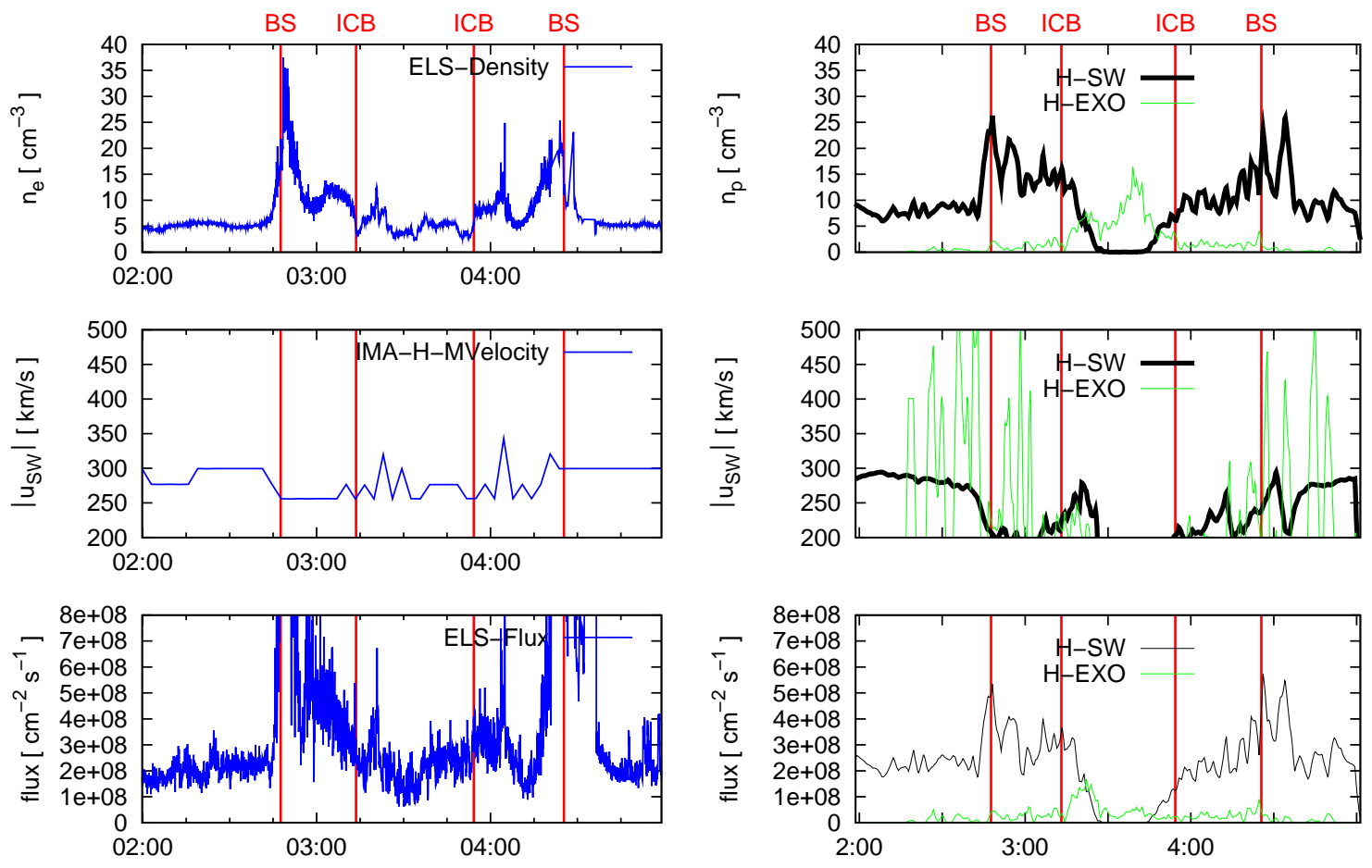

Fig. 8. Comparison of MEX/ASPERA-3 data (left) with simulation results along the MEX trajectory (right). From top to bottom: Solar wind density, proton velocity and solar wind flux. Quantities of exospheric hydrogen are presented in green.

(S. McKenna-Lawlor, private communication). The range quoted is due to uncertainties in the solar wind speed at the model's inner boundary. We then varied the angle between the IMF vector adopted and $z_{\text {MSO }}$ until best agreement was found at a value of $10^{\circ}$. Ancillary input parameter are listed in Table 2.

\subsection{Simulation results}

The simulation results are demonstrated in Fig. 7. Contour plots in the equatorial plane of the solar wind density (a) and the fluxes of ion species (b-f) are shown. The convective electric field points to the dawn hemisphere where the electric field vector is tilted by $9^{\circ}$ out of the plane. The solar wind flows around the obstacle and generates a wake in the downstream region. A bow shock is formed with a substructure called "shocklets" or "multiple shocks" which can clearly be seen in Fig. 7a. Such "shocklets" or "multiple shocks" are described respectively by Omidi and Winske (1990) and Shimazu (2001). The dense solar wind is mass-loaded due to UV ionization and by charge exchange processes, as described by Szegö et al. (2000).

Figure $7 \mathrm{~b}$ and $\mathrm{c}$ feature respectively the flux of exospheric hydrogen and oxygen. As shown in Fig. 7c the exospheric oxygen ions are accelerated away from Mars on the dawn side and in a direction toward Mars on the dusk side. Exospheric protons are deflected around the planet as shown in Fig. 7b. This different behaviors are quite coherent as the exospheric hydrogen gyroradius is about $420 \mathrm{~km}$, which is more than one order of magnitude smaller than the exospheric oxygen gyroradius and this would lead to a more fluid like behavior of hydrogen. The ionospheric ion fluxes are shown in Fig. 7d to $\mathrm{f}$. The flux values range from $10^{5}$ up to $10^{7} \mathrm{~cm}^{-2} \mathrm{~s}^{-1}$. These are in very good agreement with MEX data analyzed by Barabash et al. (2007). Immediately behind the planet the fluxes have a rather cloudy structure. These clouds move slowly between the two magnetic lobes along the plasma sheet into the tail. Similar clouds were observed at Venus by Brace et al. (1982).

\subsection{Comparison of the simulation results with MEX data}

MEX/ASPERA-3 data and simulation results are presented in Fig. 8. Both refer to the Rosetta Mars swingby. Solar wind density $n_{p}$, solar wind velocity $u_{s w}$ and solar wind flux are compared in each case. Simulated exospheric hydrogen is presented in the density plot shown in the upper right panel, in the velocity plot shown in the middle right panel and in the flux plot in the lower right panel of Fig. 8. The comparison of observed data and simulation results demonstrates a good agreement with respect to characteristic plasma structures. In particular, the positions of the bow shock and the ICB fit well. Minor discrepancies between the measurements 

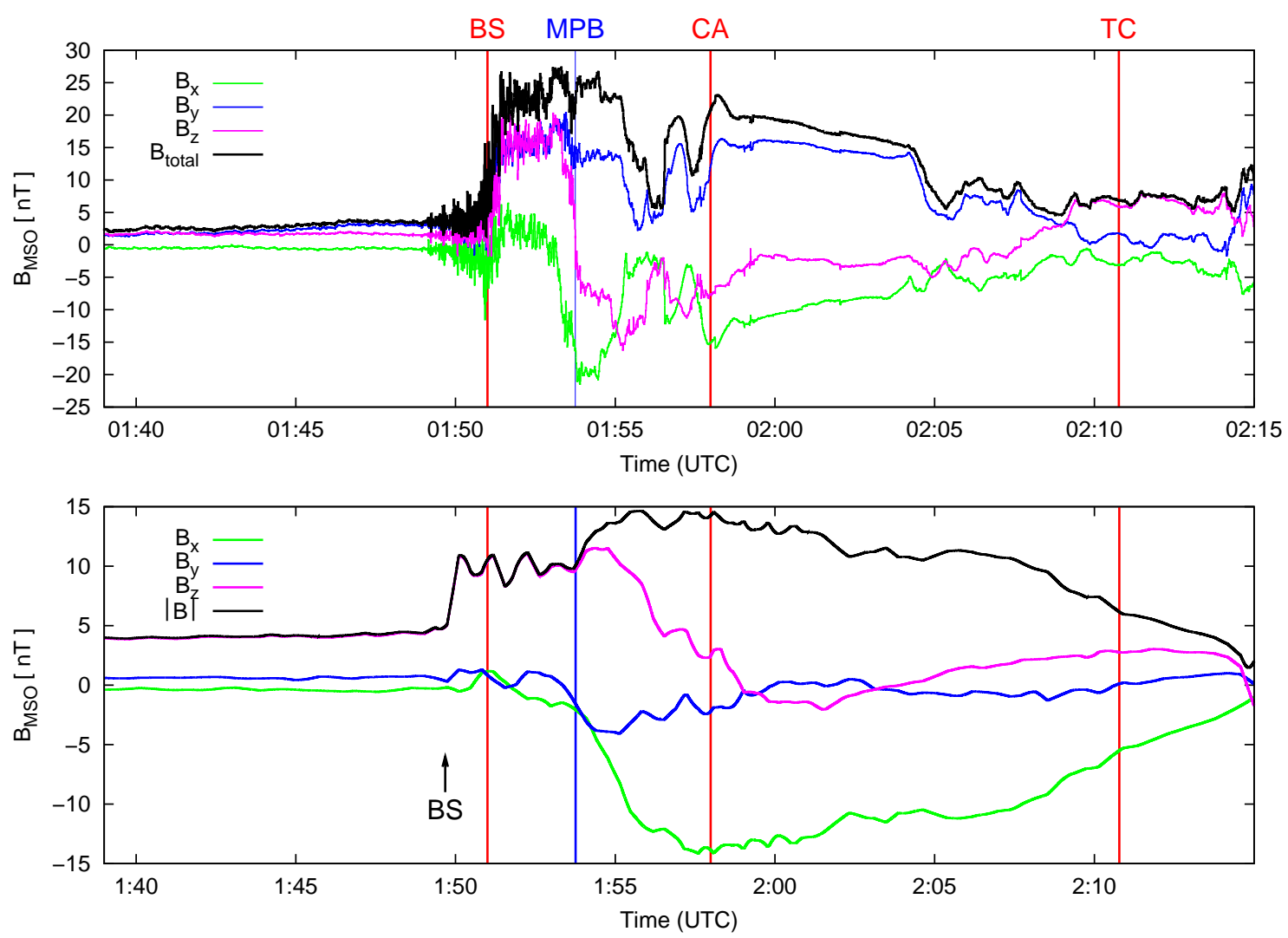

Fig. 9. ROMAP magnetic field data (top) in comparisons with results of the hybrid simulation (bottom). The hybrid model does not include effects of the crustal magnetic field which appear in the data between 01:56 UT and 01:58 UT. While Rosetta crosses the bow shock at 01:51 UT the hybrid simulation gives a bow shock position around one minute earlier. These simulation results can explain the ROMAP measurements qualitatively very well, especially the decreasing field strength in all three components at the MPB position. The simulation results produce a total magnetic field which is about $10 \mathrm{nT}$ weaker than observed values. This can be explained by numerical smoothing during the simulations and unkown magnetic field offsets for the ROMAP measurements. Furthermore on-average conditions in the solar wind do not lead to a stationar picture of Mars-solar wind interaction which is given by the simulation. Only a IMF vector with a strong positive $B_{z}$ component is able to explain the negative $B_{x}$ component during measurements behind the bow shock. For an explanation see also Fig. 10.

and simulations occur in the solar wind density. The measured electron density of the solar wind gives a value of about $5 \mathrm{~cm}^{-3}$ while the proton density varied between 2 and $10 \mathrm{~cm}^{-3}$. In the simulation a background solar wind density of $8 \mathrm{~cm}^{-3}$ was chosed. The measured peak electron density reached $35 \mathrm{~cm}^{-3}$ at the inbound BS. Numerical smoothing during the simulation reduce the density to a value of about $25 \mathrm{~cm}^{-3}$. Exospheric protons reach densities around $1 \mathrm{~cm}^{-3}$. They are accelerated by the pick-up process to high velocities of about $500 \mathrm{~km} / \mathrm{s}$. The main agreement between the measurements and the simulation results validates our choice of the input parameters of the simulation shown in Table 2. A more extensive comparison of MEX data and simulation results can be found in Bößwetter et al. (2007).

\section{Comparison of Rosetta data with simulation results}

Figure 9 shows ROMAP magnetic field data in direct comparison with hybrid simulation results. While Rosetta crossed the bow shock at 01:51 UT the hybrid simulation predicted a bow shock position at around one minute earlier. It seems that the bow shock position was shifted from the average bow shock position found by MGS (Vignes et al., 2000) just by this one minute. Thus the hybrid simulation results would agree with the average bow shock position. A short term denser or faster solar wind could lead to higher ram pressure pushing the shock inward durning the Rosetta swing-by manoeuvre.

The bow shock in the simulation results features a clear shocklet structure. The decreasing magnetic field strength in all components at the MPB position can be reproduced well by the hybrid simulation. The simulation results produce a 

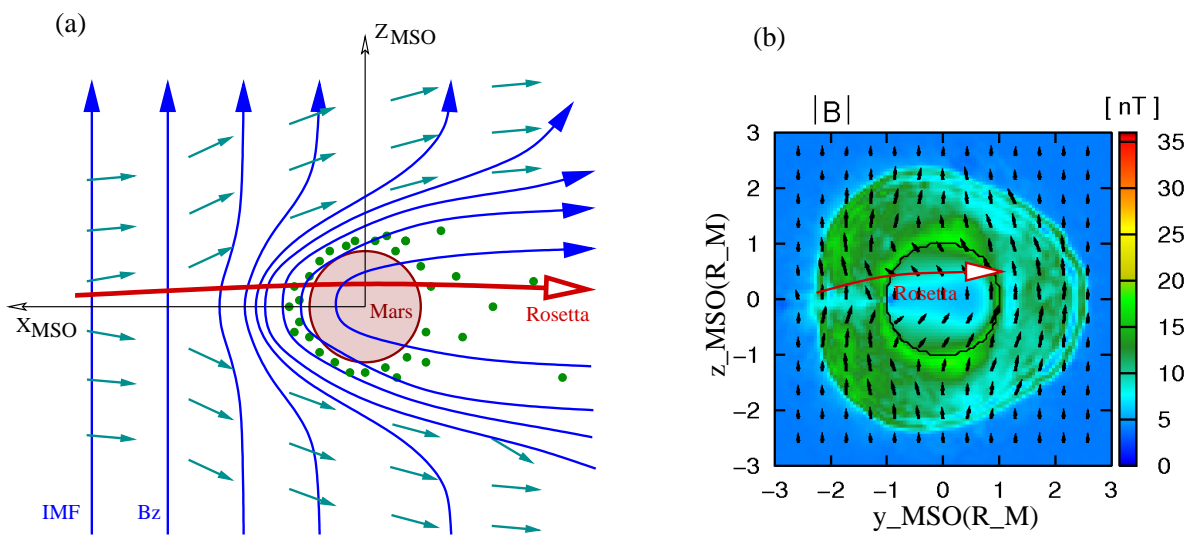

Fig. 10. Morphology of the IMF at the Rosetta swingby. (a) RPC-MAG data (see Fig. 3) as well as several hybrid simulation test runs with different IMF vector directions have shown that the IMF vector is mainly determined by its z-component to explain the measured negative $B_{x}$ component in Martian magnetosheath. (b) Simulation results of magnetic field in terminator plane in MSO coordinates. This figure explains the only slightly increase of magnetic field when Rosetta entered the MPR. In this swingby geometry Rosetta cannot cross the pronounced magnetic field increase at MPB as it would happened if Rosetta's trajectory were in the polar plane.

peak total magnetic field of about $15 \mathrm{nT}$. The observed values range up to $25 \mathrm{nT}$. This discrepancy can be explained by numerical smoothing during the simulations. As also the magnetic field offsets were unkown for the ROMAP measurements, we could not excactly determine the components of the IMF vector. The time series for the $B_{y}$ component do not show a good agreement with the data. However, after testing different offsets for $B_{y}$ component in simulations we found that here presented simulation result fits best to the measurements. Furthermore non-average conditions in the solar wind do not lead to a stationar picture of Mars-solar wind interaction which is given by the simulation. High frequency waves which are triggered by instabilities cannot be completely captured by these simulations. Further simulations with a higher grid resolution of the order of magnitude of about $10 \mathrm{~km}$ per cell behind the bow shock are necessary.

The simulation shows an important feature in Fig. 10a. Only an IMF direction with a positive $B_{z}$ component and a negligible $B_{y}$ component is able to explain the negative $B_{x}$ component during measurements in the magnetosheath, when Rosetta was above the equatorial plane.

Another question that can be addressed by the simulation results is: why do not the ROMAP field data show any jump at the MPB position? This signature depends strongly on the orbit plane of the spacecraft and the draped magnetic field pattern around the planet. While every cross-section through the magnetic lobes show a clear MPR in front of the planet and in the tail, the ideal plane between the two magnetic lobes does not show a MPR and MPB. If the IMF vector lies in the plane of the spacecraft orbit, the spacecraft will cross a magnetic lobe from the flank side. At this kind of crossing the magnetic field jump at the MPB is pronounced. ROMAP measured a piled-up magnetic field without a clear magnetic field jump at the position of the MPB. Marsis and ASPERA-3 observations onboard of MEX also reveal sometimes a transition across the MPB without magnetic field pile-up (Dubinin et al., 2008). Zhang et al. (2008) analysed magnetic field data from VEX. They found that the IMF orientation exerts a strong control on the type of the discontinuity. The boundary exhibits properties of a tangential discontinuity, while at an other IMF clock angle the boundary shows properties of a rotational discontinuity.

The magnetic field recording of the Martian MPB by the ROMAP magnetometer fits more to a rotational discontinuity. The reason for this may go back to a crossing nearly between the two magnetic lobes. It can be explained by means of Fig. 10b which shows the terminator cross-section through the simulation box. The projection of the Rosetta trajectory is also presented. In agreement with ROMAP measurements we expect from the simulation results only a slight increase in the magnetic field, when Rosetta entered the MPR. In this swingby geometry Rosetta would not cross a pronounced magnetic field increase at the MPB as would happen if the trajectory of the spacecraft were in the polar plane.

\section{Conclusions}

We present magnetic field data from a swingby manoeuvre of Rosetta at Mars. This is the first time that a spacecraft has verified the presence of the magnetic crust anomalies reported by MGS. The crustal magnetic field model by Langlais et al. (2004) is in excellent agreement with the magnetic field data, which was measured by Rosetta in ionospheric regions below around $300 \mathrm{~km}$. A detailed spectral analysis showed proton upstream waves in front of the bow shock at a frequency of $\approx 60 \mathrm{mHz}$. 
During the Rosetta Mars swingby the MEX spacecraft collected plasma data near Mars. Plasma measurements by the MEX/ASPERA-3 experiment provide additional information concerning solar wind parameters during the encounter to input to the 3-D hybrid simulations. Using these background solar wind parameters we were able to reproduce the ROMAP magnetic field measurements very well by means of our multi-ion hybrid simulations.

Simulation results produced a subsolar the subsolar bow shock at $1.5 R_{M}$ which is in good agreement with the average bow shock locations measured by MGS. ROMAP magnetic field measurements showed a slightly more compressed magnetosheath. The bow shock was pushed toward the planet, while the MPB position corresponded very well with the simulation results. We could show that the structure of the MPB depend significantly on the position of the spacecraft with respect to the magnetic field draping pattern. If the spacecraft cross the MPB in the plane between the two magnetic lobes, no magnetic field jump occur at the position of the MPB. Several simulations have shown that higher IMF strength and variations in its direction move the bow shock position only marginally. A short term denser solar wind could lead to higher ram pressure that pushing the shock inward before a higher charge exchange rate could load the solar wind more strongly with mass. Further simulation efforts will take into account a dynamical solar wind and short time scales. Several space missions are scheduled in the near future. The NASA Mars Scout Program is designed to send a series of small, low-cost missions to Mars. The Phoenix Mars Lander was the first spacecraft selected. The Mars Atmosphere and Volatile EvolutioN (MAVEN) mission will provide direct measurements in the upper atmosphere and ionosphere to answer key scientific questions about Mars' evolution.

Simultaneous atmospheric and ionospheric monitoring of the different neutral and ion species in the upper atmosphere and exosphere, through a simultaneous solar wind monitoring, as well as magnetic field measurements at low altitude are required parameters for future multi-ion hybrid simulations to investigate the interconnection between the atmosphere and variable solar wind conditions. Such measurements could be performed by the appropriate scientific payload, as suggested in another mission: the recent ESA Cosmic Vision proposal (Langlais et al., 2008; Leblanc et al., 2008). The new multi-ion simulations presented here show a statistically highly resolved exosphere that includes exospheric hydrogen. Furthermore, results of these simulations show flux values of planetary heavy ions in the same order of magnitude as those recently measured by the MEX/ASPERA-3 instrument.

Acknowledgements. This work has been supported by the Deutsche Forschungsgemeinschaft through the grants MO 539/13 and MO 539/15.

Further financial support for the work of the RPC-MAG and ROMAP Principal Investigator Team at the Technische Universitaet Braunschweig by the German Ministerium fuer Wirtschaft und
Technologie and the Deutsches Zentrum fuer Luft- und Raumfahrt under grant 50QP0402 and 50QP0201 is acknowledged.

Topical Editor I. A. Daglis thanks two anonymous referees for their help in evaluating this paper.

\section{References}

Acuña, M. H., Connerney, J. E. P., Ness, N. F., Lin, R. P., Mitchell, D., Carlson, C. W., McFadden, J., Anderson, K. A., Reme, H., Mazelle, C., Vignes, D., Wasilewski, P., and Cloutier, P.: Global Distribution of Crustal Magnetization Discovered by the Mars Global Surveyor MAG/ER Experiment, Science, 284, 790-793, 1999.

Arkani-Hamed, J.: An improved 50-degree spherical harmonic model of the magnetic field of Mars derived from both highaltitude and low-altitude data, J. Geophys. Res., 107, 5083, doi: 10.1029/2001JE001835, 2002.

Auster, H. U., Apathy, I., Berghofer, G., Remizov, A., Roll, R., Fornacon, K. H., Glassmeier, K. H., Haerendel, G., Hejja, I., Kührt, E., Magnes, W., Moehlmann, D., Motschmann, U., Richter, I., Rosenbauer, H., Russell, C. T., Rustenbach, J., Sauer, K., Schwingenschuh, K., Szemerey, I., and Waesch, R.: ROMAP: Rosetta Magnetometer and Plasma Monitor, Space Sci. Rev., 128, 221-240, doi:10.1007/s11214-006-9033-x, 2007.

Bagdonat, T. and Motschmann, U.: 3D Hybrid Simulation Code Using Curvilinear Coordinates, J. Comput. Phys., 183, 470-485, 2002 a.

Bagdonat, T. and Motschmann, U.: From a weak to a strong comet - 3D global hybrid simulation results, Earth, Moon Planets, 90, 305-321, 2002b.

Bagdonat, T., Motschmann, U., Glassmeier, K.-H., and Kührt, E.: Plasma environment of comet Churyumov-Gerasimenko 3D hybrid code simulations, in: ASSL Vol. 311: The New Rosetta Targets. Observations, Simulations and Instrument Performances, edited by: Colangeli, L., Mazzotta Epifani, E., and Palumbo, P., p. 153, 2004.

Barabash, S., Dubinin, E., Pisarenko, N., Lundin, R., and Russell, C. T.: Picked-up protons near Mars - PHOBOS observations, Geophys. Res. Lett., 18, 1805-1808, 1991.

Barabash, S., Lundin, R., Andersson, H., Brinkfeldt, K., Grigoriev, A., Gunell, H., Holmström, M., Yamauchi, M., Asamura, K., Bochsler, P., Wurz, P., Cerulli-Irelli, R., Mura, A., Milillo, A., Maggi, M., Orsini, S., Coates, A. J., Linder, D. R., Kataria, D. O., Curtis, C. C., Hsieh, K. C., Sandel, B. R., Frahm, R. A., Sharber, J. R., Winningham, J. D., Grande, M., Kallio, E., Koskinen, H., Riihelä, P., Schmidt, W., Säles, T., Kozyra, J. U., Krupp, N., Woch, J., Livi, S., Luhmann, J. G., McKenna-Lawlor, S., Roelof, E. C., Williams, D. J., Sauvaud, J.-A., Fedorov, A., and Thocaven, J.-J.: The Analyzer of Space Plasmas and Energetic Atoms (ASPERA-3) for the Mars Express Mission, Space Sci. Rev., 126, 113-164, doi:10.1007/s11214-006-9124-8, 2006.

Barabash, S., Fedorov, A., Lundin, R., and Sauvaud, J.-A.: Martian Atmospheric Erosion Rates, Science, 315, 501, doi:10.1126/ science.1134358, 2007.

Bertucci, C., Mazelle, C., Crider, D. H., Vignes, D., Acuña, M. H., Mitchell, D. L., Lin, R. P., Connerney, J. E. P., Rème, H., Cloutier, P. A., Ness, N. F., and Winterhalter, D.: Magnetic field draping enhancement at the Martian magnetic plileup boundary 
from Mars global surveyor observations, Geophys. Res. Lett., 30, 1099, doi:10.1029/2002GL015713, 2003.

Bertucci, C., Mazelle, C., and Acuña, M.: Structure and variability of the Martian magnetic pileup boundary and bow shock from MGS MAG/ER observations, Adv. Space Res., 36, 2066-2076, doi:10.1016/j.asr.2005.05.096, 2005a.

Bertucci, C., Mazelle, C., Acuña, M. H., Russell, C. T., and Slavin, J. A.: Structure of the magnetic pileup boundary at Mars and Venus, J. Geophys. Res., 110, 1209, doi:10.1029/2004JA010592, 2005b.

Bößwetter, A., Bagdonat, T., Motschmann, U., and Sauer, K.: Plasma boundaries at Mars: a 3-D simulation study, Ann. Geophys., 22, 4363-4379, 2004, http://www.ann-geophys.net/22/4363/2004/.

Bößwetter, A., Simon, S., Bagdonat, T., Motschmann, U., Fränz, M., Roussos, E., Krupp, N., Woch, J., Schüle, J., Barabash, S., and Lundin, R.: Comparison of plasma data from ASPERA3/Mars-Express with a 3-D hybrid simulation, Ann. Geophys., 25, 1851-1864, 2007, http://www.ann-geophys.net/25/1851/2007/.

Brace, L. H., Theis, R. F., and Hoegy, W. R.: Plasma clouds above the ionopause of Venus and their implications, Planet. Space Sci., 30, 29-37, 1982.

Brain, D. A.: The bow shocks and upstream waves of Venus and Mars, Adv. Space Res., 33, 1913-1919, doi:10.1016/j.asr.2003. 05.036, 2004.

Brain, D. A., Bagenal, F., Acuña, M. H., Connerney, J. E. P., Crider, D. H., Mazelle, C., Mitchell, D. L., and Ness, N. F.: Observations of low-frequency electromagnetic plasma waves upstream from the Martian shock, J. Geophys. Res., 107, 1076, doi:10.1029/ 2000JA000416, 2002.

Brain, D. A., Mitchell, D. L., and Halekas, J. S.: The magnetic field draping direction at Mars from April 1999 through August 2004, Icarus, 182, 464-473, doi:10.1016/j.icarus.2005.09.023, 2006.

Brecht, S. H.: Hybrid simulations of the magnetic topology of Mars, J. Geophys. Res., 102, 4743-4750, 1997.

Brecht, S. H. and Ledvina, S. A.: The Solar Wind Interaction With the Martian Ionosphere/Atmosphere, Space Sci. Rev., 126, 1538, doi:10.1007/s11214-006-9084-z, 2006.

Breus, T. K., Krymskii, A. M., Lundin, R., Dubinin, E. M., Luhmann, J. G., Yeroshenko, Y. G., Barabash, S. V., Mitnitskii, V. Y., Pissarenko, N. F., and Styashkin, V. A.: The solar wind interaction with Mars: consideration of Phobos-2 mission observations of an ion composition boundary on the dayside, J. Geophys. Res., 96, 11165-11174, 1991

Cain, J. C., Ferguson, B. B., and Mozzoni, D.: An $n=90$ internal potential function of the Martian crustal magnetic field, J. Geophys. Res., 108, 5008, doi:10.1029/2000JE001487, 2003.

Chaufray, J. Y., Modolo, R., Leblanc, F., Chanteur, G., Johnson, R. E., and Luhmann, J. G.: Mars solar wind interaction: Formation of the Martian corona and atmospheric loss to space, J. Geophys. Res., 112, 9009-9024, doi:10.1029/2007JE002915, 2007.

Connerney, J. E. P., Acuña, M. H., Wasilewski, P. J., Ness, N. F., Reme, H., Mazelle, C., Vignes, D., Lin, R. P., Mitchell, D. L., and Cloutier, P. A.: Magnetic Lineations in the Ancient Crust of Mars, Science, 284, 794-798, 1999.

Curtis, S. A. and Ness, N. F.: Remanent magnetism at Mars, Geophys. Res. Lett., 15, 737-739, 1988.

Dubinin, E., Modolo, R., Fraenz, M., Woch, J., Chanteur, G., Duru,
F., Akalin, F., Gurnett, D., Lundin, R., Barabash, S., Winningham, J. D., Frahm, R., Plaut, J. J., and Picardi, G.: Plasma environment of Mars as observed by simultaneous MEX-ASPERA-3 and MEX-MARSIS observations, J. Geophys. Res., 113, $10217-$ 10233, doi:10.1029/2008JA013355, 2008.

Edberg, N., Eriksson, A., Auster, U., Barabash, S., Bößwetter, A., Carr, C., Cowley, S., Cupido, E., Fränz, Glassmeier, K.-H., Goldstein, R., Leister, M., Lundin, R., Modolo, R., Nilsson, H., Richter, I., Samara, M., and Trotignon, J.: Simultaneous measurements of the Martian Plasma environment by Rosetta and Mars Express, Planet. Space Sci., in press, 2009.

Gauss, C.: Allgemeine Theorie des Erdmagnetismus., in: Resultate aus den Beobachtungen Magnetischen Vereins im Jahre 1838 pp. 1-57, Weidmann, Leipzig, Germany, translated into English by: Sabine, E., edited by: Taylor, R., in: Scientific Memoirs, vol. 2, 1841, Taylor and Taylor, pp. 184-251, London, 1839.

Glassmeier, K.-H., Boehnhardt, H., Koschny, D., Kührt, E., and Richter, I.: The Rosetta Mission: Flying Towards the Origin of the Solar System, Space Sci. Rev., 128, 1-4, doi:10.1007/ s11214-006-9140-8, 2007a.

Glassmeier, K.-H., Richter, I., Diedrich, A., Musmann, G., Auster, U., Motschmann, U., Balogh, A., Carr, C., Cupido, E., Coates, A., Rother, M., Schwingenschuh, K., Szegö, K., and Tsurutani, B.: RPC-MAG The Fluxgate Magnetometer in the ROSETTA Plasma Consortium, Space Sci. Rev., 128, 649-670, doi:10.1007/ s11214-006-9114-x, 2007b.

Hanson, W. B. and Mantas, G. P.: Viking electron temperature Measurements: Evidence for a magnetic field in the Martian ionosphere, J. Geophys. Res., 93, 7538-7544, 1988.

Israelevich, P. L., Gombosi, T. I., Ershkovich, A. I., DeZeeuw, D. L., Neubauer, F. M., and Powell, K. G.: The induced magnetosphere of comet Halley. 4. Comparison of in situ observations and numerical simulations, J. Geophys. Res., 104, 28309-28319, 1999.

Kallio, E. and Janhunen, P.: Ion escape from Mars in a quasi-neutral hybrid model, J. Geophys. Res., 107, 1035, doi:10.1029/2001JA000090, 2002.

Kallio, E., Fedorov, A., Barabash, S., Janhunen, P., Koskinen, H., Schmidt, W., Lundin, R., Gunell, H., Holmström, M., Futaana, Y., Yamauchi, M., Grigoriev, A., Winningham, J. D., Frahm, R., and Sharber, J. R.: Energisation of $\mathrm{O}^{+}$and $\mathrm{O}_{2}^{+}$Ions at Mars: An Analysis of a 3-D Quasi-Neutral Hybrid Model Simulation, Space Sci. Rev., 126, 39-62, doi:10.1007/s11214-006-9120-z, 2006.

Krasnopolsky, V. A.: Mars' upper atmosphere and ionoshere at low, medium, and high solar activities: Implications for evolution of water, J. Geophys. Res., 107, 5128, doi:10.1029/2001JE001809, 2002.

Langlais, B., Purucker, M. E., and Mandea, M.: Crustal magnetic field of Mars, J. Geophys. Res., 109, 2008, doi:10.1029/ 2003JE002048, 2004.

Langlais, B., Leblanc, F., Fouchet, T., Barabash, S., Breuer, D., Chassefière, E., Coates, A., Dehant, V., Forget, F., Lammer, H., Lewis, S., Lopez-Valverde, M., Mandea, M., Menvielle, M., Pais, A., Paetzold, M., Read, P., Sotin, C., Tarits, P., Vennerstrom, S., Branduardi-Raymont, G., Cremonese, G., Merayo, J. G. M., Ott, T., Rème, H., Trotignon, J. G., and Walhund, J. E.: Mars Environment and Magnetic Orbiter: model payload, Experimental Astronomy, pp. 38-61, doi:10. 1007/s10686-008-9101-1, 2008. 
Leblanc, F., Langlais, B., Fouchet, T., Barabash, S., Breuer, D., Chassefière, E., Coates, A., Dehant, V., Forget, F., Lammer, H., Lewis, S., Lopez-Valverde, M., Mandea, M., Menvielle, M., Pais, A., Paetzold, M., Read, P., Sotin, C., Tarits, P., and Vennerstrom, S.: Mars Environment and Magnetic Orbiter: Science and Measurement Objectives, Astrobiology, doi:10.1089/AST.2007. 022, 2008.

Liu, Y., Nagy, A. F., Gombosi, T. I., DeZeeuw, D. L., and Powell, K. G.: The solar wind interaction with Mars: results of threedimensional three-species MHD studies, Adv. Space Res., 27, 1837-1846, 2001.

Ma, Y., Nagy, A. F., Hansen, K. C., DeZeeuw, D. L., Gombosi, T. I., and Powell, K. G.: Three-dimensional multispecies MHD studies of the solar wind interaction with Mars in the presence of crustal fields, J. Geophys. Res., 107, 1282-1289, 2002.

Ma, Y.-J. and Nagy, A. F.: Ion escape fluxes from Mars, Geophys. Res. Lett., 34, 8201, doi:10.1029/2006GL029208, 2007.

McKenna-Lawlor, S. M. P., Dryer, M., Fry, C. D., Sun, W., Lario, D., Deehr, C. S., Sanahuja, B., Afonin, V. A., Verigin, M. I., and Kotova, G. A.: Predictions of energetic particle radiation in the close Martian environment, J. Geophys. Res., 110, 3102, doi: 10.1029/2004JA010587, 2005.

Modolo, R., Chanteur, G. M., Dubinin, E., and Matthews, A. P.: Influence of the solar EUV flux on the Martian plasma environment, Ann. Geophys., 23, 433-444, 2005,

http://www.ann-geophys.net/23/433/2005/.

Modolo, R., Chanteur, G. M., Dubinin, E., and Matthews, A. P.: Simulated solar wind plasma interaction with the Martian exosphere: influence of the solar EUV flux on the bow shock and the magnetic pile-up boundary, Ann. Geophys., 24, 3403-3410, 2006, http://www.ann-geophys.net/24/3403/2006/.

Moehlmann, D.: The question of a Martian planetary magnetic field, Adv. Space Res., 12, 213-217, doi:10.1016/0273-1177(92) 90391-A, 1992.

Motschmann, U. and Kührt, E.: Interaction of the Solar Wind with Weak Obstacles: Hybrid Simulations for Weakly Active Comets and for Mars, Space Sci. Rev., 122, 197-207, doi: 10.1007/s11214-006-6218-2, 2006.

Omidi, N. and Winske, D.: Steepening of kinetic magnetosonic waves into shocklets: Simulations and consequences for planetary shocks and comets, J. Geophys. Res., 95, 2281-2300, 1990.

Purucker, M., Ravat, D., Frey, H., Voorhies, C., Sabaka, T., and Acuña, M.: An altitude-normalized magnetic map of Mars and its interpretation, Geophys. Res. Lett., 27, 2449-2452, doi:10. 1029/2000GL000072, 2000.

Russell, C. T., Luhmann, J. G., Schwingenschuh, K., Riedler, W., and Yeroshenko, Y.: Upstream waves at Mars - PHOBOS observations, Geophys. Res. Lett., 17, 897-900, 1990.

Sauer, K. and Dubinin, E.: The nature of the Martian "obstacle boundary”, Adv. Space Res., 26, 1633-1637, 2000.
Sauer, K., Bogdanov, A., and Baumgärtel, K.: Evidence of an ion composition boundary (protonopause) in bi-ion fluid simulations of solar wind mass loading, Geophys. Res. Lett., 21, 2255-2258, 1994.

Schwenn, R.: Large-Scale Structure of the Interplanetary Medium, in: Physics of the Inner Heliosphere - Part One - Largescale Phenomena, vol. 20 of Physics and Chemistry in Space - Space and Solar Physics, pp. 99-181, Springer-Verlag, 1990.

Shimazu, H.: Three-dimensional hybrid simulation of solar wind interaction with unmagnetized planets, J. Geophys. Res., 106, 8333-8342, 2001.

Simon, S., Bagdonat, T., Motschmann, U., and Glassmeier, K.-H.: Plasma environment of magnetized asteroids: a 3-D hybrid simulation study, Ann. Geophys., 24, 407-414, 2006, http://www.ann-geophys.net/24/407/2006/.

Simon, S., Boesswetter, A., Bagdonat, T., and Motschmann, U.: Physics of the Ion Composition Boundary: a comparative 3-D hybrid simulation study of Mars and Titan, Ann. Geophys., 25, 99-115, 2007a, http://www.ann-geophys.net/25/99/2007/.

Simon, S., Boesswetter, A., Bagdonat, T., Motschmann, U., and Schuele, J.: Three-dimensional multispecies hybrid simulation of Titan's highly variable plasma environment, Ann. Geophys., 25, 117-144, 2007b, http://www.ann-geophys.net/25/117/2007/.

Simon, S., Motschmann, U., Kleindienst, G., Glassmeier, K.-H., Bertucci, C., and Dougherty, M. K.: Titan's magnetic field signature during the Cassini T34 flyby: Comparison between hybrid simulations and MAG data, Geophys. Res. Lett., 35, 4107, doi: 10.1029/2007GL033056, 2008.

Stebbings, R. F., Smith, A. C. H., and Ehrhardt, H.: Charge Transfer between Oxygen Atoms and $\mathrm{O}+$ and H+ Ions, J. Geophys. Res., 69, 2349-2355, 1964.

Szegö, K., Glassmeier, K.-H., Bingham, R., Bogdanov, A., Fischer, C., Haerendel, G., Brinca, A., Cravens, T., Dubinin, E., Sauer, K., Fisk, L., Gombosi, T., Schwadron, N., Isenberg, P., Lee, M., Mazelle, C., Möbius, E., Motschmann, U., Shapiro, V. D., Tsurutani, B., and Zank, G.: Physics of Mass Loaded Plasmas, Space Sci. Rev., 94, 429-671, 2000.

Trotignon, J. G., Dubinin, E., Grard, R., Barabash, S., and Lundin, R.: Martian planetopause as seen by the plasma wave system onboard Phobos 2, J. Geophys. Res., 101, 24965-24977, 1996.

Vignes, D., Mazelle, C., Rme, H., Acuña, M. H., Connerney, J. E. P., Lin, R. P., Mitchell, D. L., Cloutier, P., Crider, D. H., and Ness, N. F.: The Solar Wind interaction with Mars: locations and shapes of the Bow Shock and the observations of the MAG/ER experiment onboard Mars Global Surveyor, Geophys. Res. Lett., 27, 49-52, 2000.

Zhang, T. L., Delva, M., Baumjohann, W., Volwerk, M., Russell, C. T., Wei, H. Y., Wang, C., Balikhin, M., Barabash, S., Auster, H.-U., and Kudela, K.: Induced magnetosphere and its outer boundary at Venus, J. Geophys. Res., 113, 20-29, doi: 10.1029/2008JE003215, 2008. 